\title{
Radiation-associated allograft injury in kidney transplant recipients with urothelial carcinoma
}

\author{
Sheng-Ping Hung ${ }^{1}$, Yang-Jen Chiang ${ }^{2}$, Ji-Hong Hong ${ }^{1}$, Kang-Hsing Fan ${ }^{1,3}$ \\ ${ }^{1}$ Department of Radiation Oncology, Proton and radiation therapy center, Linkou Chang Gung Memorial Hospital, Taoyuan, Taiwan; ${ }^{2}$ Division \\ of Urology, Department of Surgery, Linkou Chang Gung Memorial Hospital, Taoyuan, Taiwan; ${ }^{3}$ Department of Radiation Oncology, New Taipei \\ Municipal TuCheng Hospital, Chang Gung Memorial Hospital and Chang Gung University, Taoyuan City, Taiwan \\ Contributions: (I) Conception and design: KH Fan; (II) Administrative support: All author; (III) Provision of study material or patients: YJ Chiang, JH \\ Hong, KH Fan; (IV) Collection and assembly of data: SP Hung; (V) Data analysis and interpretation: SP Hung; (VI) Manuscript writing: All authors; \\ (VII) Final approval of manuscript: All authors. \\ Correspondence to: Kang-Hsing Fan, MD, Department of Radiation Oncology, New Taipei Municipal TuCheng Hospital, Chang Gung Memorial \\ Hospital and Chang Gung University, No.5, Fuxing St., Guishan Dist., Taoyuan City 33305, Taiwan, R.O.C. Email: khs.fan@gmail.com.
}

Background: This retrospective study was conducted to evaluate the safety of radiotherapy for kidney transplant recipients (KTRs) with urothelial carcinoma (UC).

Methods: KTRs who developed UC and received radiotherapy with or without chemotherapy at our hospital from 1995 to 2016 were identified as the study group. All patients were treated by 3-dimensional conformal radiotherapy (3D-CRT) or intensity-modulated radiation therapy (IMRT). Various chemotherapy protocols were applied according to each patient's risk factors. The primary end point was graft failure. The tolerance dose of kidney was defined according to the QUANTEC report for evaluation. The cause of graft failure was determined by a kidney transplantation specialist.

Results: The study group comprised ten patients with a median age of 63 years, and their diagnoses included 3 upper urinary tract UC, 4 bladder cancers, 3 synchronous UC in both the upper and lower urinary tracts. Half of the patients were treated by 3D-CRT, and the others were treated by IMRT. Dose distribution of only 2 treatment plans violated the tolerance dose of kidney (V6Gy $<30 \%$ ), but neither of them induced renal graft failure. There were four renal graft failures in the study group, which were due to different causes, including recurrent infection, second primary UC, and chronic allograft nephropathy.

Conclusions: We suggest that the current irradiation technique is safe for kidney allograft when treating UC and can comply almost all the constraints suggested by the QUANTEC report. Following the QUANTEC report to constrain the radiation dose delivered to the kidney can be safe to avoid renal function impairment in KTRs.

Keywords: Transitional cell carcinoma; kidney transplantation (KT); radiotherapy

Received: 02 July 2019; Accepted: May 28 2020; Published: 30 September 2020.

doi: $10.21037 /$ tro-19-71

View this article at: http://dx.doi.org/10.21037/tro-19-71

\section{Introduction}

Kidney transplantation (KT) is known as renal replacement therapy that is performed in patients with end-stage renal disease and results in better survival and quality of life than dialysis $(1,2)$. However, the survival of kidney transplant recipients (KTRs) is significantly affected by rejection (3), due to which the Kidney Disease: Improving Global Outcomes (KDIGO) guideline has suggested long-term use of immunosuppressants (4). Nevertheless, immunosuppressant use may increase the incidence of different types of cancer resulting from the modulation of the immune system or an increase in the risk of viral 
infection (5). In contrast to data from western countries, which reported that squamous cell carcinoma of skin is the cardinal malignancy after KT and account for $40 \%$ cases, previous studies from Wu et al. (6) have also demonstrated that urothelial carcinoma (UC) is the leading cause of malignancies in KTRs with an incidence which is $4.1 \%$ in Taiwan. The ratio between observed and expected case number was 398.4. Besides higher incidence of UC in KTRs, Chiang et al. (7) also found a different pattern with female predominance and higher incidence of upper tract involvement. The clinical course of UC in KTRs is usually aggressive (6) and may have poor overall survival (8).

In patients with newly diagnosed bladder cancer, concurrent chemoradiotherapy (CCRT) is recommended as an organ-preserving modality for those with muscle invasive bladder cancer or those who are not suitable for surgery (9). Radiotherapy (RT) alone is also indicated for those who are too old or too weak to receive surgery and chemotherapy $(10,11)$. CCRT or RT alone has also been used as an adjuvant or salvage setting for patients with UC having a high risk for local failure or localregional recurrence after surgery. The clinical target volume (CTV) would include pelvic lymph nodes with/ without the entire bladder for bladder cancer (12) and also include the entire urinary tract and pelvic lymph nodes in case of upper urinary tract UC (13). Therefore, the transplanted kidney that is generally implanted in the pelvis would also be exposed to the radiation dose.

However, there is a lack of studies on the radiation dose distribution and its effects on allograft function in KTRs with UC. Moreover, it is also not clear whether the tolerance radiation dose of KTRs is similar to that of subjects with normal kidney. Therefore, this study was conducted to explore the impact of modern RT on these patients. We present the following article in accordance with the STROBE reporting checklist (available at http:// dx.doi.org/10.21037/tro-19-71) (14).

\section{Method}

\section{Patient group, primary and secondary end point}

We retrospectively searched for KTRs who had urinary tract UC and were followed up in our hospital from 1995/1/1 to $2015 / 12 / 31$. All patients who had received pelvic RT were identified and their charts and treatment plans were reviewed from the first day of RT until 2019/05/31. We collected all the information regarding underlying diseases, renal transplant causes, immunosuppressant agents, cancer treatment modalities, and laboratory data related to renal function (including urinary analysis, hemogram, and biochemical markers). All patients had been followed up by the transplantation specialist. The primary end point was radiation-related allograft failure, which was defined as an irreversible renal function decline that ended up in another renal replacement therapy if the patient received overdose radiation and had no other definite reason identified after complete workup. The secondary endpoint were local control and regional control. Local control was defined as no recurrent disease occurring in urinary tract within the radiation field. Regional control was defined as no relapsed regional lymph node metastasis during follow up. The graft survival, disease control and overall survival time was calculated from the first day of RT until the day of index event, such as graft failure, disease recurrent, patient mortality or censored by using Kaplan-Meier method. The study was conducted in accordance with the Declaration of Helsinki (as revised in 2013) and was approved by Chang Gung Medical Foundation Institutional Review Board (IRB): 201700191B0. The IRB approved the waiver of the informed consent form. A copy of the IRB certification is available for review by the Editor-in-Chief of this journal.

\section{Radiation and chemotherapy}

All patients were treated by CT-based planning, and 3-dimensional RT or intensity-modulated radiation therapy (IMRT) was used. Since some of these patients had received more than one RT session, we summed up all treatment plans to evaluate the dose applied to the allograft in these patients. Target contouring, treatment planning, and dose computing were operated using Eclipse ${ }^{\mathrm{TM}}$ Treatment Planning System, Ver. 8.6 and Ver. 13 (Varian Medical System, Palo Alto, CA, USA), sequentially. We used the tolerance dose suggested by the QUANTEC report for evaluation (15). The reason for graft failure was reviewed by the transplant specialist if the end point was identified. The transplant specialist also determined whether the radiation induced graft failure, especially for patients whose graft kidney received excess radiation dose.

Various chemotherapy protocols were applied, such as adjuvant therapy and concurrent chemotherapy, according to the patient's risk factors and underlying comorbidities. 


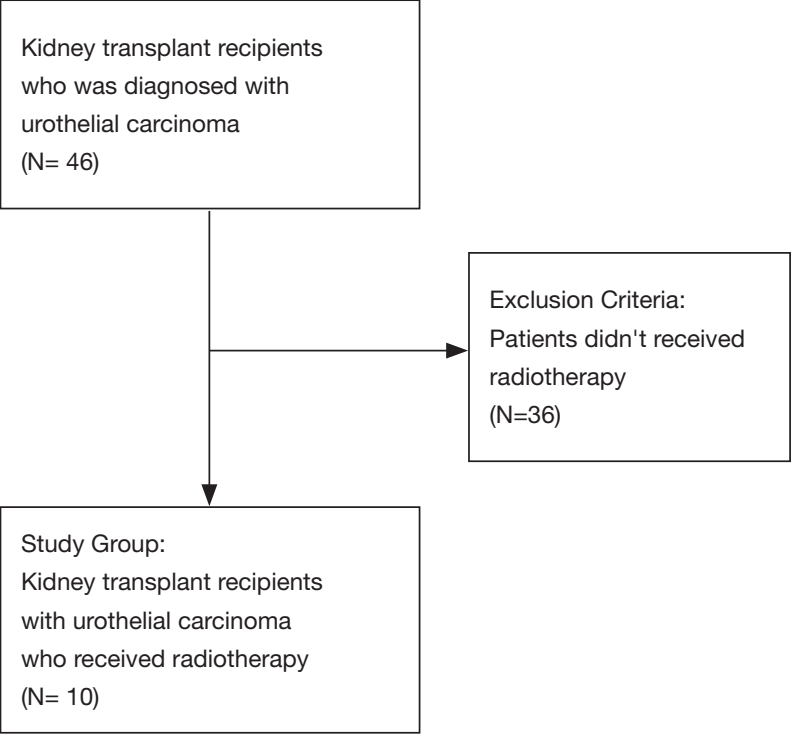

Figure 1 Flow diagram.

\section{Results}

\section{Patient characteristics and outcomes}

During follow up period, there were 46 KTRs diagnosed with UC and received treatment in our hospital. After excluding patients who didn't receive RT, a total of 10 KTRs were included as study group (Figure 1). The study group comprised 5 males and 5 females with a median age of 63 years, who developed UC had received RT from 2005 to 2016. The median follow-up time was 42.9 months (range: 15.3-165.2 months). Three patients had upper urinary tract $\mathrm{UC}$, four had bladder $\mathrm{UC}$, and the remaining three patients had synchronous UC in both the upper and lower urinary tracts. Six of these patients received RT as adjuvant therapy after surgery, and the remaining four received RT as a part of bladder preservation treatment. Six patients were treated with RT alone, and the others were treated with concurrent chemotherapy. All the concurrent chemotherapy was cisplatin-based regiment. One patient received weekly Cisplatin and the other three received Cisplatin/Fluorouracil/Leucovorin. Three of the ten patients received second irradiation, including two who received reirradiation for a $2^{\text {nd }}$ primary $\mathrm{UC}$ and one patient who received treatment for parascrotal metastases. After treatment, there were two local failures, two regional failures, and six distant metastases. There were also six patients who had new primary UC, which was another
$\mathrm{UC}$ occurred in contralateral side of urinary tract or in the same side but happened in another organ. The 5-year local control, regional control, distant metastasis-free, and overall survival rates were $90 \%, 80 \%, 60 \%$, and $70 \%$, respectively. Neither local control nor regional control resulted in graft failure. New primary UC was the only oncologic event resulting in graft failure.

The median graft survival period was 34.97 months after RT. Four KTRs developed graft failure, including two patients associated with tumor progression/recurrent, which results in obstructive uropathy and upper urinary tract infection. The third graft failure was due to recurrent pyelonephritis, and the final one was related to chronic allograft nephropathy. Table 1 summarizes the characteristics and outcomes of all study patients.

The mode of treatment included $50 \%$ of 3 -dimensional conformal RT (3D-CRT) and $50 \%$ of IMRT. The mean dose applied to the allograft was 3.69 Gy ( 0.52-8.6 Gy, SD $=2.43 \mathrm{~Gy})$. Pre-radiation creatinine clearance ranged from 33.22 to $73.66 \mathrm{~mL} / \mathrm{min}$. Since we used the tolerance dose suggested in the QUANTEC report for evaluation, we observed that the renal allografts of two patients were exposed to a radiation dose exceeding QUANTEC's suggestion. These two grafts had extremely low-dose irradiated volume and broke the V6Gy $<30 \%$ constraint. Tables 2,3 show the details about RT such as the CTV, graft location, and dose volume results. Acute gastrointestinal (GI) complications were observed in 8 patients, including 1 grade 2 diarrhea, 6 grade 1 diarrhea, and 1 grade 1 constipation. There were also seven patients with genitourinary (GU) toxicities, including 3 grade 2 urine frequency, 1 grade 1 urine frequency, and 1 grade 1 hematuria. Severe acute toxicities or late complications were not detected.

\section{A case with graft failure and excess radiation dose}

One of the patients whose graft received excessive radiation developed graft failure. This patient was a 64-year-old female who received KT in 1989. She had also been diagnosed with new-onset diabetes after transplantation (NODAT) before she developed UC. Bladder UC was diagnosed in 2009, and she received repeated TURBT. Hydronephrosis of graft kidney was observed during the follow-up, and thus, cancer recurrence in the bladder was noted in 2012. She received partial cystectomy with graft ureterectomy in 2012. The graft ureter was invaded and 
Table 1 Patient characteristics and outcomes

\begin{tabular}{|c|c|}
\hline & $\mathrm{N}(\%)$ \\
\hline Age (median age, range) & $63.50(40-74)$ \\
\hline \multicolumn{2}{|l|}{ Sex } \\
\hline Female & $5(50.0 \%)$ \\
\hline Male & $5(50.0 \%)$ \\
\hline \multicolumn{2}{|l|}{ Smoking } \\
\hline No & $8(80.0 \%)$ \\
\hline Yes & $2(20.0 \%)$ \\
\hline \multicolumn{2}{|l|}{ Hypertension } \\
\hline No & $1(10.0 \%)$ \\
\hline Yes & 9 (90.0\%) \\
\hline \multicolumn{2}{|l|}{ Diabetes mellitus } \\
\hline No & $8(80.0 \%)$ \\
\hline Yes & $2(20.0 \%)$ \\
\hline \multicolumn{2}{|l|}{ Cancer location } \\
\hline Upper urinary tract & $3(30.0 \%)$ \\
\hline Bladder & $4(40.0 \%)$ \\
\hline Both & $3(30.0 \%)$ \\
\hline \multicolumn{2}{|l|}{$\mathrm{T}$} \\
\hline $\mathrm{T} 1$ & $1(10.0 \%)$ \\
\hline T2 & $4(40.0 \%)$ \\
\hline T3 & $3(30.0 \%)$ \\
\hline T4 & $2(20.0 \%)$ \\
\hline \multicolumn{2}{|l|}{$\mathrm{N}$} \\
\hline No & $9(90.0 \%)$ \\
\hline N1 & $0(00.0 \%)$ \\
\hline N2 & $1(10.0 \%)$ \\
\hline N3 & $0(00.0 \%)$ \\
\hline \multicolumn{2}{|l|}{ M } \\
\hline MO & $9(90.0 \%)$ \\
\hline M1 & $1(10.0 \%)$ \\
\hline \multicolumn{2}{|l|}{ Treatment modality } \\
\hline RT alone & $6(60.0 \%)$ \\
\hline CCRT & $4(40.0 \%)$ \\
\hline \multicolumn{2}{|l|}{ Radiotherapy method } \\
\hline 3D-CRT & $5(50.0 \%)$ \\
\hline
\end{tabular}

Table 1 (continued)
Table 1 (continued)

\begin{tabular}{|c|c|}
\hline & $\mathrm{N}(\%)$ \\
\hline IMRT & $5(50.0 \%)$ \\
\hline \multicolumn{2}{|l|}{ Graft location } \\
\hline Outfield & $3(30.0 \%)$ \\
\hline Infield & $7(70.0 \%)$ \\
\hline Mean dose & $3.69(0.52-8.6)$ \\
\hline \multicolumn{2}{|l|}{ Graft failure } \\
\hline No & $6(60.0 \%)$ \\
\hline Yes & $4(40.0 \%)$ \\
\hline \multicolumn{2}{|c|}{ Hydronephrosis } \\
\hline No & $7(70.0 \%)$ \\
\hline Yes & $3(30.0 \%)$ \\
\hline \multicolumn{2}{|c|}{ Chronic allograft nephropathy } \\
\hline No & $8(80.0 \%)$ \\
\hline Yes & $2(20.0 \%)$ \\
\hline \multicolumn{2}{|l|}{ Recurrent } \\
\hline No & $3(30.0 \%)$ \\
\hline Yes & $6(60.0 \%)$ \\
\hline Persistent & $1(10.0 \%)$ \\
\hline \multicolumn{2}{|c|}{ Distant metastasis } \\
\hline No & $4(40.0 \%)$ \\
\hline Yes & $6(60.0 \%)$ \\
\hline \multicolumn{2}{|l|}{ Expire } \\
\hline No & $7(70.0 \%)$ \\
\hline Yes & $3(30.0 \%)$ \\
\hline
\end{tabular}

the stage was rpT3aN0M0. Postoperative adjuvant pelvic RT was administered due to positive resection margin. She received image-guided IMRT to the tumor bed and pelvic lymph nodes from March 15, 2013, to May 4, 2013. The mean dose applied to the graft kidney was 8.6 Gy. After the completion of RT, chemotherapy with fluorouracil, leucovorin, and cisplatin was administered every 2 months from May 2013 to April 2014. Meanwhile, she also received gemcitabine every 2 weeks. All mentioned chemotherapies were suspended in case severe hematological side effects or a decline in renal function was observed. The patient's renal function declined progressively since September 2013. Renal echo suggested chronic pyelonephritis during the follow-up. 
Table 2 Radiotherapy information

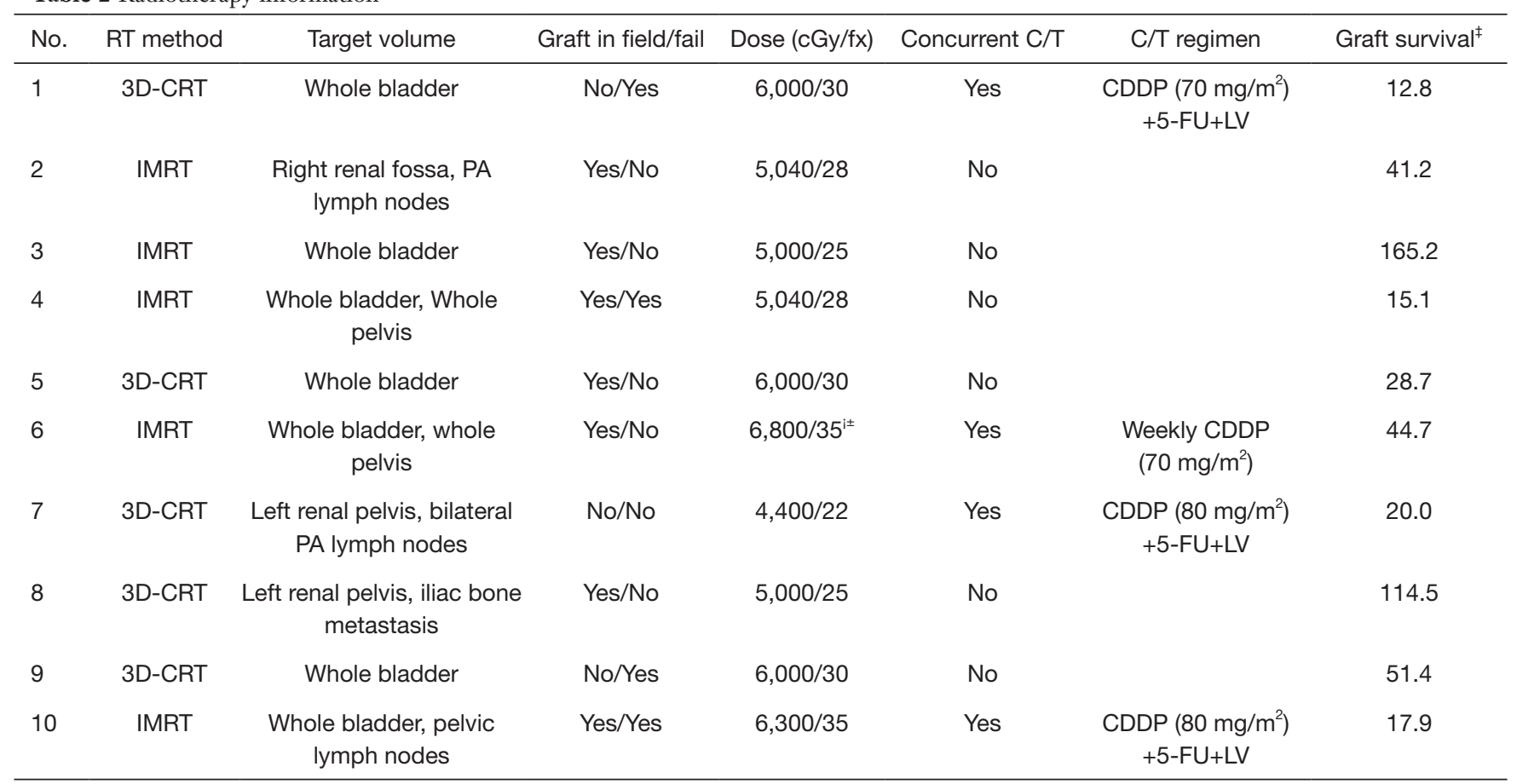

${ }^{\S}$ pelvic lymph nodes $5,040 \mathrm{cGy} / 28 \mathrm{fx}$, then reduced field to whole bladder to 6,300 cGy/35fx with residual tumor boost to 6,800 cGy. ${ }^{\ddagger}$ graft survival (months): graft survival from last day of RT to graft failure day or the day case censored. PA, paraaortic; CDDP, Cisplatin; 5-FU, fluorouracil; LV, leucovorin.

Table 3 Radiation dose applied to the kidney graft

\begin{tabular}{|c|c|c|c|c|c|c|}
\hline No. & Mean dose $(G y)^{\dagger}$ & V28 $(\%)^{\dagger}$ & V23 $(\%)^{\dagger}$ & V20 $(\%)^{\dagger}$ & V12 $(\%)^{\dagger}$ & V6 $(\%)^{\dagger}$ \\
\hline 2 & 5.51 & 4.54 & 6.07 & 7.28 & 12.38 & 23.25 \\
\hline 3 & 2.64 & 0 & 0 & 0.14 & 3.84 & 19.29 \\
\hline 4 & 1.8 & 0.54 & 1.33 & 2.09 & 4.13 & 6.6 \\
\hline 6 & 4.98 & 0 & 0 & 0 & 0 & 40 \\
\hline 7 & 0.52 & 0 & 0 & 0 & 0 & 0 \\
\hline 8 & 3.79 & 0 & 0 & 0 & 0 & 0 \\
\hline 9 & 2.43 & 0 & 0 & 0 & 0 & 0 \\
\hline
\end{tabular}

${ }^{\dagger}$ Suggested dose constraint by QUANTAC: mean dose <18 Gy, V28<20\%, V23<30\%, V20<32\%, V12<55\%, V6 <30\% (remaining kidney).

She was hospitalized due to recurrent urinary tract infection from April 2014 to August 2014, and she also developed renal failure in September 2014 for which she received hemodialysis since then. The KT specialist suggested that
RT played a minor role in this patient's allograft failure because the patient had already developed hydronephrosis before receiving RT, and the hydronephrosis didn't improve after treatment even after percutaneous nephrostomy (PCN) 

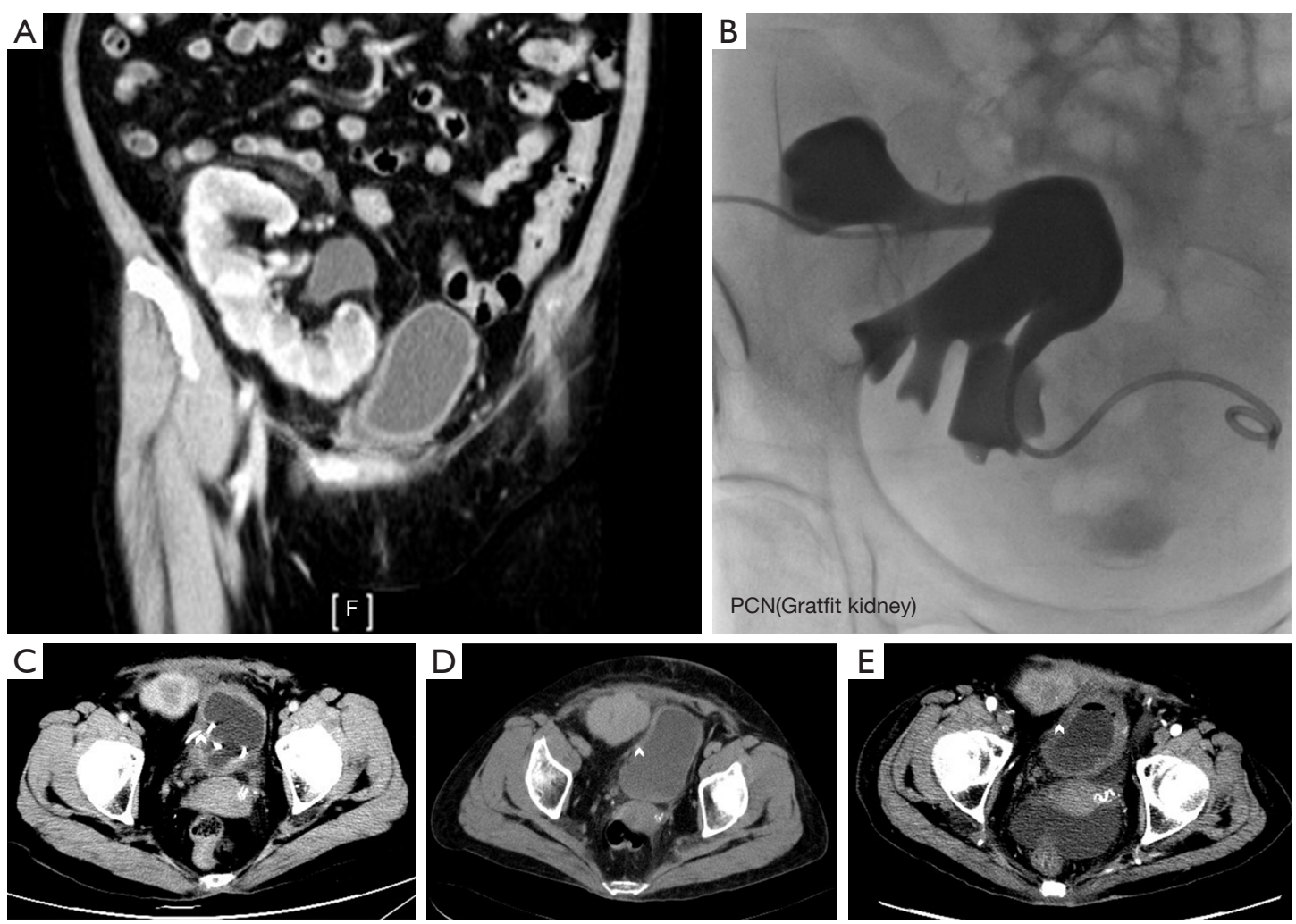

Figure 2 Follow up study for patient 10. (A) Image study in 4 months after complete RT, which showed graft kidney hydronephrosis. (B) PCN revision in 12 months after RT. The hydronephrosis was persisted even after PCN. (C) Suspicious UVJ lesion just after complete RT. (D) UVJ lesion in 4 months after RT. (E) Progressive change of the UVJ lesion in 12 months after RT. Arrowhead: Suspicious UVJ lesion. UVJ, ureterovesical junction; RT, radiotherapy; PCN, percutaneous nephrostomy.

during follow up (Figure $2 A, B)$. We also found that there was a suspicious residual lesion near ureterovesical junction (UVJ) been found in the first image study after RT (Figure $2 C$ ), and the lesion regressed after adjuvant chemotherapy (Figure 2D) but it became more prominent during follow up (Figure 2E). The persistent hydronephrosis may result from the UVJ lesion. In addition, she developed recurrent urinary tract infection that required parenteral antibiotics and was admitted for 2 months just before the graft failure. In conclusion, it was more likely that patient's graft function progressively declined because of recurrent lesion and the acute infection deteriorated the process. However, we could not find any tissue proof of this patient retrospectively.

\section{Discussion}

Based on our study results, there were no radiation-related graft failures. The dose-volume histogram analysis revealed that most of the patients ( 8 of 10$)$ were within the constraint suggested by the QUANTAC report and no patient had graft failure caused by radiation after reviewing by the KT specialist. Patients violated the QUANTAC constraint because the graft received excess low-dose irradiation (V6 $>30 \%$ ). One of them had graft failure, but it was not radiation-related. In addition, the other three graft failures received only little radiation dose (mean dose $=1.87 \mathrm{~Gy}$ ). Moreover, these patients did not develop severe acute or late toxicity when we strictly reduced the radiation dose to the kidney. Neither the GI nor the GU tract developed complications greater than grade 3. Therefore, we concluded that the current technique, 3D-CRT and IMRT, for RT can have a good dose-sparing effect for the kidney graft without increasing treatment toxicities and can be used as a safe treatment modality for KTRs with UC.

According to the Scientific Registry of Transplant Recipients (SRTR) \& Organ Procurement and Transplant Network (OPTN) 2017 annual report, the rate of graft failures has been increasing continuously and is 
approximately half of what it was 10 years ago (16). The acute graft failure rate was $4.3 \%$ in the report; however, the graft failure rate still increased with time from approximately $20 \%$ in 3 years to $30 \%$ in 5 years after transplantation (17). This so-called late graft failure would make patients return to dialysis, which is presumed to increase mortality (18). In this case series, the graft failure rate was $40 \%$, and the median graft survival period was 8.9 years after transplantation. This result was comparable to previous data reported from European and American patients, wherein the 10 -year graft survival rate was $56 \%$ for European and 45.7\% for American patients (19).

Radiation can also affect renal function because it results in radiation nephropathy. The definite pathogenesis remains unclear till date, and the current explanation is that radiation would injure the glomerular endothelium and the tubular epithelium and cause interstitial scarring (20). Clinical symptoms appear months or years after RT, which include proteinuria, anemia, elevated serum creatinine, urine casts, and hypertension. Dawson et al. (15) suggested that tolerance to RT reduced in patients with one kidney whose condition is just similar to that of our study group, i.e., the KTRs.

However, there is no literature discussing radiation toxicity in patients with UC with KT. A previous research on prostate cancer conducted by Mouzin et al. (21) reviewed the effect of 3D-CRT and reported that external beam RT with a dose of up to 70 Gy in 2 Gy per fraction applied to prostate cancer could achieve adequate cancer control at the expense of infraclinical ureteral obstruction. The graft function was not directly affected by radiation because the CTV covered only the prostate without pelvic irradiation and the graft kidney was away from treatment field. However, the CTV in our study was larger than that reported in these studies, which included the prostate plus a $10-\mathrm{mm}$ margin in the report from Mouzin et al. (21). In addition, the CTV covered the upper pelvic cavity or the lower abdominal region, where the graft kidney was located, thus the graft might receive considerable radiation dose. Pelvic irradiation in KTRs has been discussed in a retrospective study $(22)$ and two case reports $(23,24)$. Although the results suggested that it is feasible to administer pelvic irradiation to KTRs by 3D-CRT (22) or IMRT $(23,24)$, the patients in the historical study were those with cervical cancer, prostate cancer, anal cancer. They were different from patients with UC. UC can directly cause urinary/renal function impairment by developing obstruction or secondary urinary traction or pyelonephritis. This implies that the renal function of KTRs is more fragile once they develop UC. In the current study, the mean dose applied to the graft kidney was $3.69 \mathrm{~Gy}$, which is much lower than that suggested by QUANTEC, i.e., a mean dose of $<18$ Gy, using 3D-CRT or IMRT. Moreover, no radiation-induced graft failures were identified. Based on these results, we suggest that it is feasible to treat $\mathrm{UC}$ in KTRs using the current RT technique and following the QUANTEC constraints.

On the other hand, the major cause of graft failure in our series was cancer-related complications, which included direct invasion by recurrent tumor and severe infection after the development of new primary $\mathrm{UC}$ in the renal pelvis. According to Chiang et al. (7) and Wu et al. (6), UC in KTRs has a more aggressive presentation and more upper urinary tract invasion and tend to have multiple foci. These features may contribute to a higher risk of direct invasion of graft or obstructive uropathy, which could result in worse graft function. This situation was also found in the current study. Half of the patients were diagnosed with multiple involvement, and $60 \%$ of them developed metachronous tumors during the follow-up. The 5-year local control and regional control rates were $90 \%$ and $80 \%$, respectively. It is difficult to compare our results with published data from the non-KTR group because of the small case number and the different patient comorbidities. However, we can still suggest that RT is an effective treatment based on the current results. Although the treatment is effective, oncologic events are still the primary reason for graft failure in these patients. This should be a consequence of the nature of the disease, multiple metachronous tumors, which may result from field cancerization. There were $>50 \%$ of patients in the study group with new primary UC. A regular follow-up with a cystoscope, CT, MRI, and urine cytology is highly suggested.

There are two major limitations in our study. The first one is the retrospective study design and the small case number. Moreover, we focused on patients who had received RT as adjuvant therapy. This may be due to the fact that the study group is rare, which could cause some selection bias. Second, only one patient with graft failure underwent biopsy before graft failure, which was because there were definite reasons that could be confirmed without biopsy. However, it was impossible to define whether there was post-RT change without pathological examination and the explanation was based on clinical evidence only. 


\section{Conclusions}

This study included 10 patients who had post-KT UC and received RT since 2005 . The median graft survival was comparable to previous data, but the leading cause of graft failure was cancer-related, which is unlike that in normal population and may result from the vicious presentation of UC in KTRs. No radiation-induced graft failure was found. This result suggests that using IMRT or 3D-CRT for treating these patients could have a good organ-sparing effect and that RT was relatively safe for these patients when the radiation dose applied to the graft followed the constraints of the QUANTEC report. Therefore, there is no need to omit RT for KTRs with UC.

\section{Acknowledgments}

Funding: None.

\section{Footnote}

Reporting Checklist: The authors have completed the STROBE reporting checklist. Available at http://dx.doi. org/10.21037/tro-19-71

Conflicts of Interest: All authors have completed the ICMJE uniform disclosure form (available at http://dx.doi. org/10.21037/tro-19-71). The authors have no conflicts of interest to declare.

Ethical Statement: The authors are accountable for all aspects of the work in ensuring that questions related to the accuracy or integrity of any part of the work are appropriately investigated and resolved. The study was conducted in accordance with the Declaration of Helsinki (as revised in 2013) and was approved by Chang Gung Medical Foundation Institutional Review Board (IRB): 201700191B0. The IRB approved the waiver of the informed consent form. A copy of the IRB certification is available for review by the Editor-in-Chief of this journal.

Open Access Statement: This is an Open Access article distributed in accordance with the Creative Commons Attribution-NonCommercial-NoDerivs 4.0 International License (CC BY-NC-ND 4.0), which permits the noncommercial replication and distribution of the article with the strict proviso that no changes or edits are made and the original work is properly cited (including links to both the formal publication through the relevant DOI and the license). See: https://creativecommons.org/licenses/by-nc-nd/4.0/.

\section{References}

1. Wolfe RA, Ashby VB, Milford EL, et al. Comparison of mortality in all patients on dialysis, patients on dialysis awaiting transplantation, and recipients of a first cadaveric transplant. N Engl J Med 1999;341:1725-30.

2. Knoll G, Cockfield S, Blydt-Hansen T, et al. Canadian Society of Transplantation: consensus guidelines on eligibility for kidney transplantation. CMAJ 2005;173:S1-25.

3. Kim SJ, Lee HH, Lee DS, et al. Prognostic factors affecting graft and patient survival in cadaveric and living kidney transplantation. Transplant Proc 2004;36:2038-9.

4. Kidney Disease: Improving Global Outcomes Transplant Work G. KDIGO clinical practice guideline for the care of kidney transplant recipients. Am J Transplant 2009;9 Suppl 3:S1-155.

5. Vajdic CM, McDonald SP, McCredie MR, et al. Cancer incidence before and after kidney transplantation. JAMA 2006;296:2823-31.

6. Wu MJ, Lian JD, Yang CR, et al. High cumulative incidence of urinary tract transitional cell carcinoma after kidney transplantation in Taiwan. Am J Kidney Dis 2004;43:1091-7.

7. Chiang YJ, Yang PS, Wang HH, et al. Urothelial cancer after renal transplantation: an update. Transplant Proc 2012;44:744-5.

8. Ardelt PU, Rieken M, Ebbing J, et al. Urothelial Cancer in Renal Transplant Recipients: Incidence, Risk Factors, and Oncological Outcome. Urology 2016;88:104-10.

9. Network. NCC. Bladder Cancer Version 1.2017. 2016. Available online: https://www.nccn.org/professionals/ physician_gls/pdf/bladder.pdf.

10. Wujanto C, Tey J, Chia D, et al. Radical radiotherapy in older patients with muscle invasive bladder cancer. J Geriatr Oncol 2019;10:292-7.

11. Fonteyne V, Ost P, Bellmunt J, et al. Curative Treatment for Muscle Invasive Bladder Cancer in Elderly Patients: A Systematic Review. Eur Urol 2018;73:40-50.

12. Baumann BC, Bosch WR, Bahl A, et al. Development and Validation of Consensus Contouring Guidelines for Adjuvant Radiation Therapy for Bladder Cancer After Radical Cystectomy. Int J Radiat Oncol Biol Phys 2016;96:78-86.

13. Chen B, Zeng ZC, Wang GM, et al. Radiotherapy 
may improve overall survival of patients with T3/T4 transitional cell carcinoma of the renal pelvis or ureter and delay bladder tumour relapse. BMC Cancer 2011;11:297.

14. von Elm E, Altman DG, Egger M, et al. The Strengthening the Reporting of Observational Studies in Epidemiology (STROBE) Statement: Guidelines for Reporting Observational Studies. PLoS Med 2007;4:e296.

15. Dawson LA, Kavanagh BD, Paulino AC, et al. Radiationassociated kidney injury. Int J Radiat Oncol Biol Phys 2010;76:S108-15.

16. Hart A, Smith JM, Skeans MA, et al. OPTN/SRTR 2017 Annual Data Report: Kidney. Am J Transplant 2019;19 Suppl 2:19-123.

17. Matas AJ, Smith JM, Skeans MA, et al. OPTN/SRTR 2012 Annual Data Report: kidney. Am J Transplant 2014;14 Suppl 1:11-44.

18. Brar A, Markell M, Stefanov DG, et al. Mortality after Renal Allograft Failure and Return to Dialysis. Am J Nephrol 2017;45:180-6.

19. Gondos A, Dohler B, Brenner H, et al. Kidney graft

doi: $10.21037 /$ tro-19-71

Cite this article as: Hung SP, Chiang YJ, Hong JH, Fan KH. Radiation-associated allograft injury in kidney transplant recipients with urothelial carcinoma. Ther Radiol Oncol 2020;4:12. survival in Europe and the United States: strikingly different long-term outcomes. Transplantation 2013;95:267-74.

20. Cohen EP, Robbins ME. Radiation nephropathy. Semin Nephrol 2003;23:486-99.

21. Mouzin M, Bachaud JM, Kamar N, et al. ThreeDimensional Conformal Radiotherapy for Localized Prostate Cancer in Kidney Transplant Recipients. Transplantation 2004;78:1496-500.

22. Dahlke S, Schwarz A, Bruns F, et al. Pelvic radiotherapy after renal transplantation. Anticancer Res 2012;32:5083-6.

23. Mohiuddin MM, Mahmood U, Hall AA, et al. Adjuvant pelvic irradiation for cervical cancer in the setting of a transplanted pelvic kidney. J Cancer Res Ther 2012;8:427-9.

24. Rosenfelder N, Corbett R, Long M, et al. Intensity modulated radiation therapy allows prostate and doseescalated pelvic radical radiation therapy after renal transplantation. Pract Radiat Oncol 2015;5:e207-13. 